\title{
Glutamate neurocircuitry: theoretical underpinnings in schizophrenia
}

\author{
Thomas L. Schwartz ${ }^{1}$, Shilpa Sachdeva ${ }^{1}$ and Stephen M. Stahl ${ }^{2,3}$ \\ 1 Department of Psychiatry, State University of New York Upstate Medical University, Syracuse, NY, USA \\ ${ }^{2}$ Department of Psychiatry, University of California San Diego, San Diego, CA, USA \\ ${ }^{3}$ Cambridge University, Cambridge, UK
}

\section{Edited by:}

Nick Andrews, Harvard Medical

School, USA

Reviewed by:

Brian H. Harvey, North-West

University, South Africa

Jason B. Wu, Cedars-Sinai Medical

Center, USA

*Correspondence:

Thomas L. Schwartz, Department of Psychiatry, State University of New York Upstate Medical University, 750 East Adams Street, Syracuse, NY

13210, USA.

e-mail: schwartt@upstate.edu
The Dopamine Hypothesis of Schizophrenia is actively being challenged by the NMDA Receptor Hypofunctioning Hypothesis of Schizophrenia. The latter hypothesis may actually be the starting point in neuronal pathways that ultimately modifies dopamine pathways involved in generating both positive and negative symptoms of schizophrenia postulated by the former hypothesis. The authors suggest that even this latter, NMDA receptor-based, hypothesis is likely too narrow and offer a review of typical glutamate and dopamine-based neurocircuitry, propose genetic vulnerabilities impacting glutamate neurocircuitry, and provide a broad interpretation of a possible etiology of schizophrenia. In conclusion, there is a brief review of potential schizophrenia treatments that rely on the etiologic theory provided in the body of the paper.

Keywords: schizophrenia, dopamine hypothesis, glutamate hypothesis, NMDA receptor

\section{INTRODUCTION}

Hypotheses for the origin of schizophrenia symptoms have likely moved further than the original "Dopamine Hypothesis" where it is postulated that overactive mesolimbic dopamine (DA) neurons cause the positive symptoms of psychosis and the corollary that underactive mesocortical DA neurons, cause the negative, $\operatorname{cog}$ nitive, and affective symptoms of schizophrenia. For more than 30 years, this key hypothesis has dominated theories of schizophrenia and is placed prominently in every psychiatric textbook written today. This theory was reverse engineered based initially upon observations that drugs that increase DA, such as amphetamine and cocaine, can create psychotic symptoms, whereas antipsychotic drugs that decrease DA by antagonizing dopamine D2 receptors actually diminish psychotic symptoms (Meltzer and Stahl, 1976). Figure 1 displays the overactivity of mesolimbic DA circuitry as the basis for development of positive symptoms, and Figure 2 depicts the hypofunctioning of the mesocortical DA pathway which projects to the frontal cortex (Meltzer and Stahl, 1976; Stahl, 2007a). This hypofrontality is the proposed mediator of negative, cognitive, and affective symptoms of schizophrenia.

The Dopamine Hypothesis of Schizophrenia has been accepted as fact and antipsychotic medications have continued to be developed based upon the mechanism of antagonizing D2 receptors in hopes of lowering the firing and activity of the mesolimbic DA pathway. Clinicians from the 1950s onward were able to use the typical antipsychotics that were first developed from phenothiazine chemical structures and then utilized other chemical classes, i.e., butyrophenones, thioxanthenes, etc., as antipsychotic agents. In the 1990s, the atypical antipsychotics were developed, branded, and marketed with a dual serotonin-dopamine receptor antagonism (SDA) mechanism of action whereby they simultaneously block both D2 and serotonin-2A (5HT-2A) receptors allowing for adequate antipsychotic effectiveness while lowering the risk of extrapyramidal syndromes (EPS). This improved neuromuscular safety profile occurs as the 5HT-2A receptor antagonism allows these novel agents to be more selective at dampening mesolimbic DA activity while allowing less interference in the nigrostriatal DA pathway (Stahl, 2007a; Opler and Opler, 2012).

Interestingly, from a clinician's point of view it is not often asked, "where did the excess dopamine activity come from?" Simplistically, a schizophrenic might have too much DA production, too little catabolism, too active or sensitive D2 receptors, etc. Schizophrenia therefore was postulated to be an illness based upon the Stress-Diathesis Model (Sadock and Sadock, 2003; Straub and Weinberger, 2006) where an individual likely has inherited one or more genes that code for abnormal proteins, and these proteins likely modify the way the mesolimbic DA pathway operates. The net result is that these abnormal proteins, i.e., receptors, enzymes, etc. likely impact upon the mesolimbic DA system making it hyperactive resulting in psychotic symptom development (Stahl, 2007a). This diathesis, or biological risk, also has to be paired with environmental stress to create enough symptoms to warrant the syndromal diagnosis of schizophrenia.

If schizophrenia developed only out of the handful of DA related genes in the human genome, then researchers should be able to create much more consistently and fully effective drugs for treating positive and negative symptoms. After 60 years of research, there are now likely safer antipsychotic medications, but they have failed to become more effective overall. Thinking outside of the box would suggest that there has to be more to the pathology of schizophrenia than just DA neurons that project from the ventral tegmental area (VTA) to the limbic structures of the brain. Perhaps, elevated mesolimbic DA activity creates one form, or type 

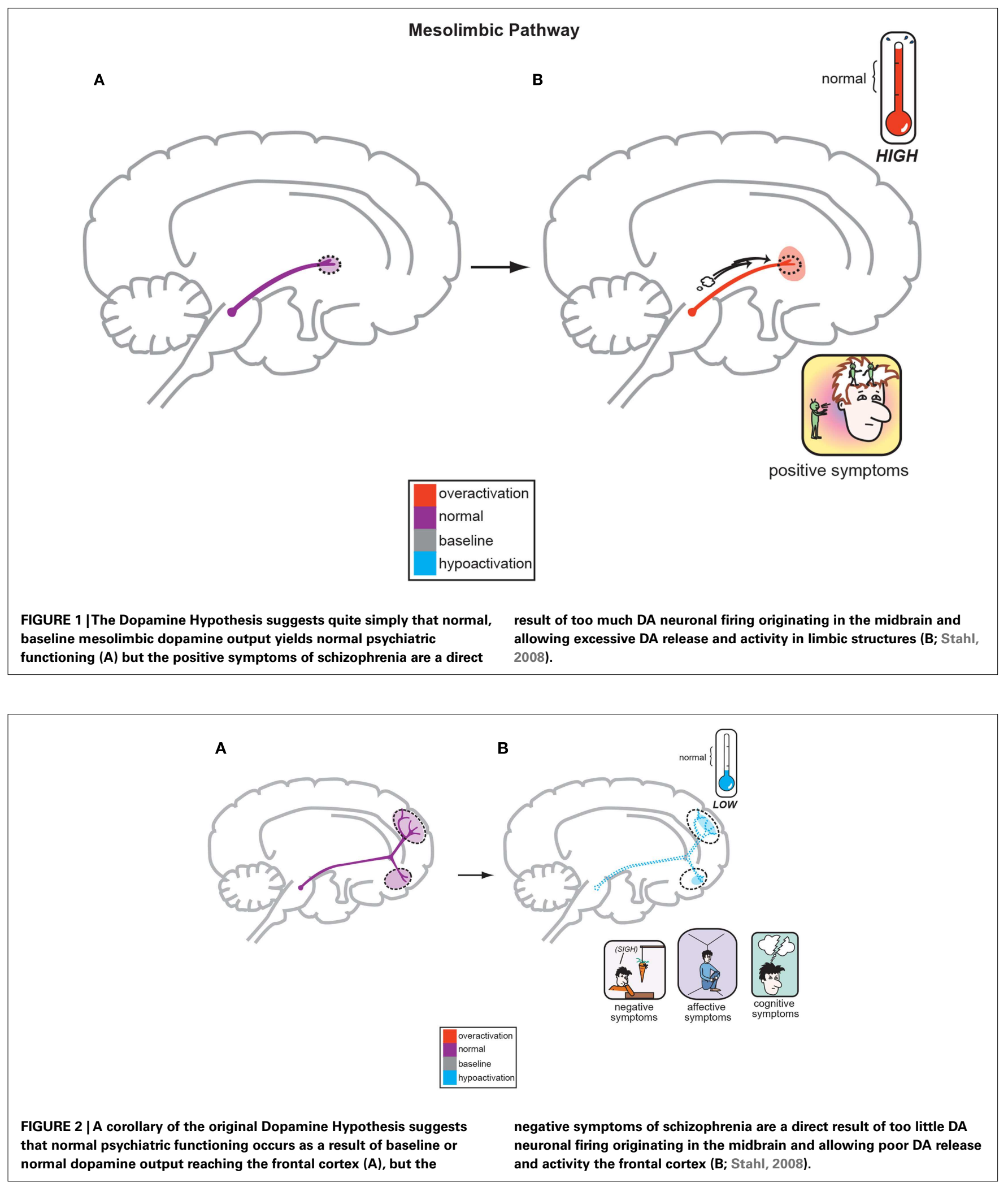

of schizophrenia? Patients with "dopamine sensitive schizophrenia" can be cured and have symptom remission on the available antipsychotic agents. Clinically, like a patient with breast cancer who undergoes genetic testing to determine if her cancer is estrogen sensitive or not, a schizophrenic could be genetically analyzed to see where his genetic vulnerability lies. If this diathesis happens 
to be in the DA mesolimbic system, then he statistically should respond very well to any of the marketed antipsychotics. If this schizophrenic patient is not positive for DA risk genes, then he is likely to have treatment resistant or refractory schizophrenia when treated with the available antipsychotic agents.

The oversimplification dictated by the Dopamine Hypothesis and the less than stellar clinical remission outcomes with D2 receptor antagonizing antipsychotics would suggest that either DA hyperactivity is only one part of the etiology and onset of schizophrenia, or perhaps it is the final common pathway whereby stress and multiple other neurotransmitters, receptors, neuronal pathways, etc. have to become jeopardized and converge on the mesolimbic system allowing DA hyperactivity to finally ensue. It is possible that some schizophrenia patients have normal DA pathways and their symptoms originate in other neuroanatomic structures that function primarily under the influence of non-DA neurotransmitters.

In this manner, the NMDA Receptor Hypofunction Hypothesis, has garnered much research and writing. Here, a faulty series of NMDA glutamate (GLU) receptors located on gamma aminobutyric acid (GABA) interneurons are purported to ultimately allow the generation of excessive mesolimbic DA activity outlined in the original Dopamine Hypothesis (Stahl, 2007a). This GLU variable now increases the etiologic complexity regarding development of schizophrenia symptoms as there is now interplay between GLU, GABA, and DA neurotransmitters. There is also neuroanatomic complexity in that the GLU neurons originate in the frontal cortex but descend into the limbic structures to exert their control over DA functioning. Now, to develop schizophrenia, a patient might have to inherit vulnerable genes in the DA, GLU, or even GABA-based neuronal systems. This fact would indicate that new approaches geared toward developing new treatments for schizophrenia would necessarily have to begin a divergence away from mastering D2 receptor antagonism pharmacodynamically, and move toward agents with other mechanistic properties.

This paper will now focus, not only on the NMDA Receptor Hypofunctioning Hypothesis of schizophrenia, but more globally investigate how a faulty glutamatergic system may impact neuropsychiatric function and possibly be related to the onset of positive and negative symptoms of schizophrenia.

\section{NEUROANATOMIC PRIMER: GLUTAMATE, GABA, AND DOPAMINE CIRCUITS}

The rest of this paper and understanding of its theories necessitates understanding some basic neuronal pathways in a very simplistic form. Figure 3 depicts a series of GLU neurons that begin in

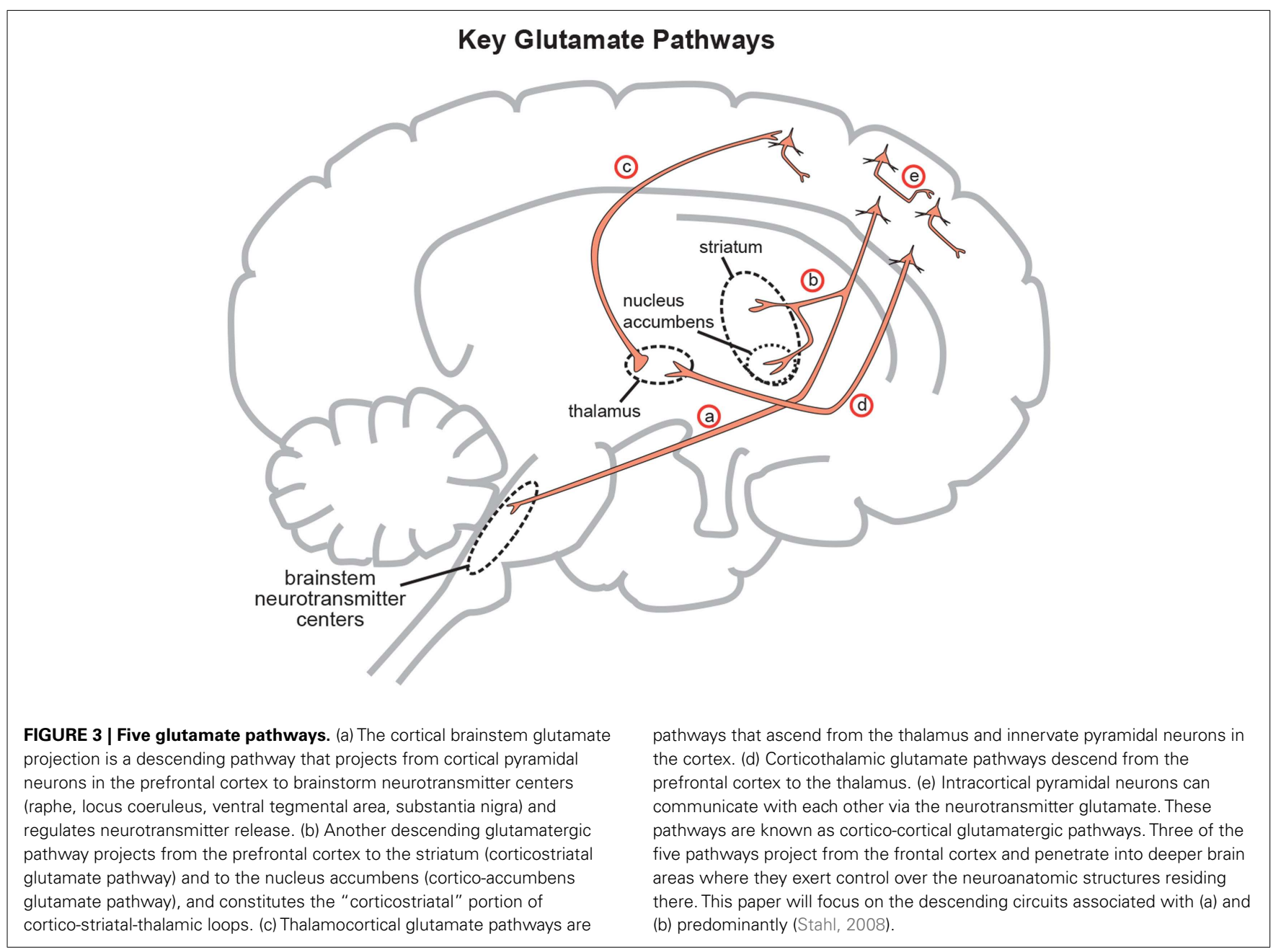


the frontal cortex and connect and project in to brainstem, midbrain, and limbic areas. In this way, neurons originating in the more modern frontal neocortex may penetrate into deeper areas of the brain to exert control over midbrain centers that are primarily responsible for creating and projecting neurotransmitter activity that are ultimately responsible for drive and affective initiation. These primary GLU neurons may project further to deeper brain areas such as the amygdala and nucleus accumbens creating appropriate perceptual balance versus psychosis. This simple circuitry will be the basis for understanding the onset of negative and positive symptoms of schizophrenia.

In order to focus on the development of positive psychotic symptoms, the cortical brainstem glutamate projection (Figure 3 (a)) must be examined more closely in its normal functioning state (Figure 4A) and its abnormal state (Figure 4B) as far as schizophrenia symptom development is concerned.

As outlined in Figures 4A,B below, DA neuronal activity originating in the midbrain and limbic structures does not exist in a vacuum and is likely controlled by GLU neurons in the frontal cortex to a great extent. Strong cortical primary neuronal GLU firing creates a circuit where strong GABA interneuron tone next occurs thus dampening secondary cortical GLU neuronal tone. This loss of GLU tone which arrives at the origin of the DA mesolimbic pathway can lower DA neuronal firing which is actually the normal state (homeostasis) and allows for normal psychiatric functioning. The excess DA from the Dopamine Hypothesis might actually be derived from the GLU neurocircuitry system. The NMDA Receptor Hypofunctioning Hypothesis suggests that NMDA receptors attached to the GABA interneurons situated between the primary and secondary GLU cortical neurons are to blame. These defective, insensitive NMDA receptors do not receive adequate stimulation from the primary GLU neuron thus making the GABA interneuron less effective, firing less often. This loss of GABA output onto the secondary GLU neuron allows it to fire more often, directly causing the firing of more and excessive DA (the Dopamine Hypothesis) neurons in the mesolimbic pathway resulting in psychotic symptoms. This makes the GLU-GABA-GLU-DA circuit complete, but now abnormal in functioning. The theoretical neuroanatomic circuitry involved in the Dopamine Hypothesis and the Glutamate Hypothesis has previously been briefly reviewed by one of the authors (Stahl, 2007a,b, 2008) and the genetic underpinnings of the Glutamate Hypothesis by both (Schwartz et al., 2012). Further

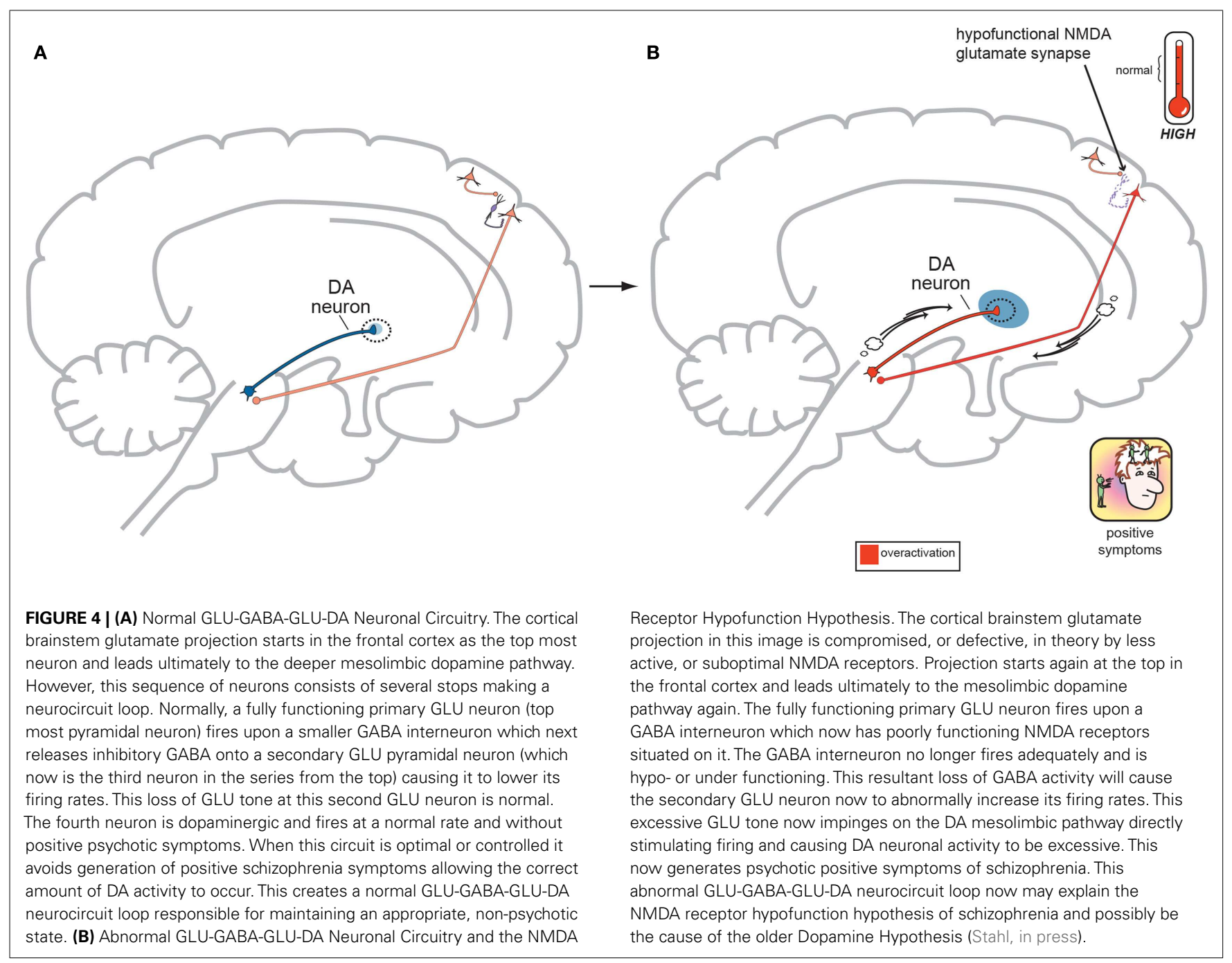


in this paper, the authors will try to merge all accounts in order to allow the reader to think through the possible genetic origins of schizophrenia symptoms and how these phenotypic symptoms may emerge at the onset of this severe and persistent psychiatric disorder.

In regards to the negative symptoms of schizophrenia, another GLU neurocircuit must be examined (Figure 3). The cortical brainstem glutamate projection has sub-circuits that are less direct and do involve two series of GABA interneurons that impact upon VTA DA neurons whose purpose it is to ascend back to the dorsolateral prefrontal cortex (DLPFC) and ventromedial prefrontal cortex (VMPFC). These circuits should provide sufficient activity for alertness, concentration, emotional, and executive functioning. These abilities are often lost, or deficient in schizophrenia, and are noted as being negative symptoms (Figure 2). A loss of DA tone in this circuitry or a change in GLU tone that drives the final DA pathway may create underactivity and inefficient performance in the DLPFC/VMPFC allowing negative symptoms to develop (Figure 5). Theoretically, if a patient has inherited genes which code for abnormal proteins that impact by lowering initial GLU tone or activity in these circuits, then patients may become hypofrontal with less cortical DA activity and negative symptoms could develop as well. This type of circuit could become defective due to abnormal GLU NMDA receptors sitting on GABA interneurons, or any other impingement on the circuit that would result in final DA common pathway changes further downstream.

In conclusion for this first section, the basic dopamine and glutamate neuropathways have been reviewed in order to alert the reader as to the current, and most accepted, individual theories behind the development of schizophrenia symptoms. They have been simplified and by using adult learning techniques (visuospatial processing by use of Figures, planned redundancy instead of rote memorization, mnemonics, associations between textsfigures-verbal constructs, etc.) ideally will be easier to recall and recognize factually. Later in this paper, these circuits will need to be remembered and referred to in order to understand how genetic findings and protein abnormalities might lead to the dysfunction of these neurocircuits and development of schizophrenia symptoms. For the more advanced, neuroscience or neuroanatomic reader, this simple theoretical material may be made more complex by reviewing the extensive work by Carlsson et al. (1999) and the following figures may represent the greater complexity of the neuroanatomic basis of the dopaminergic and glutamatergic hypotheses of schizophrenia (see Figures 6 and 7).

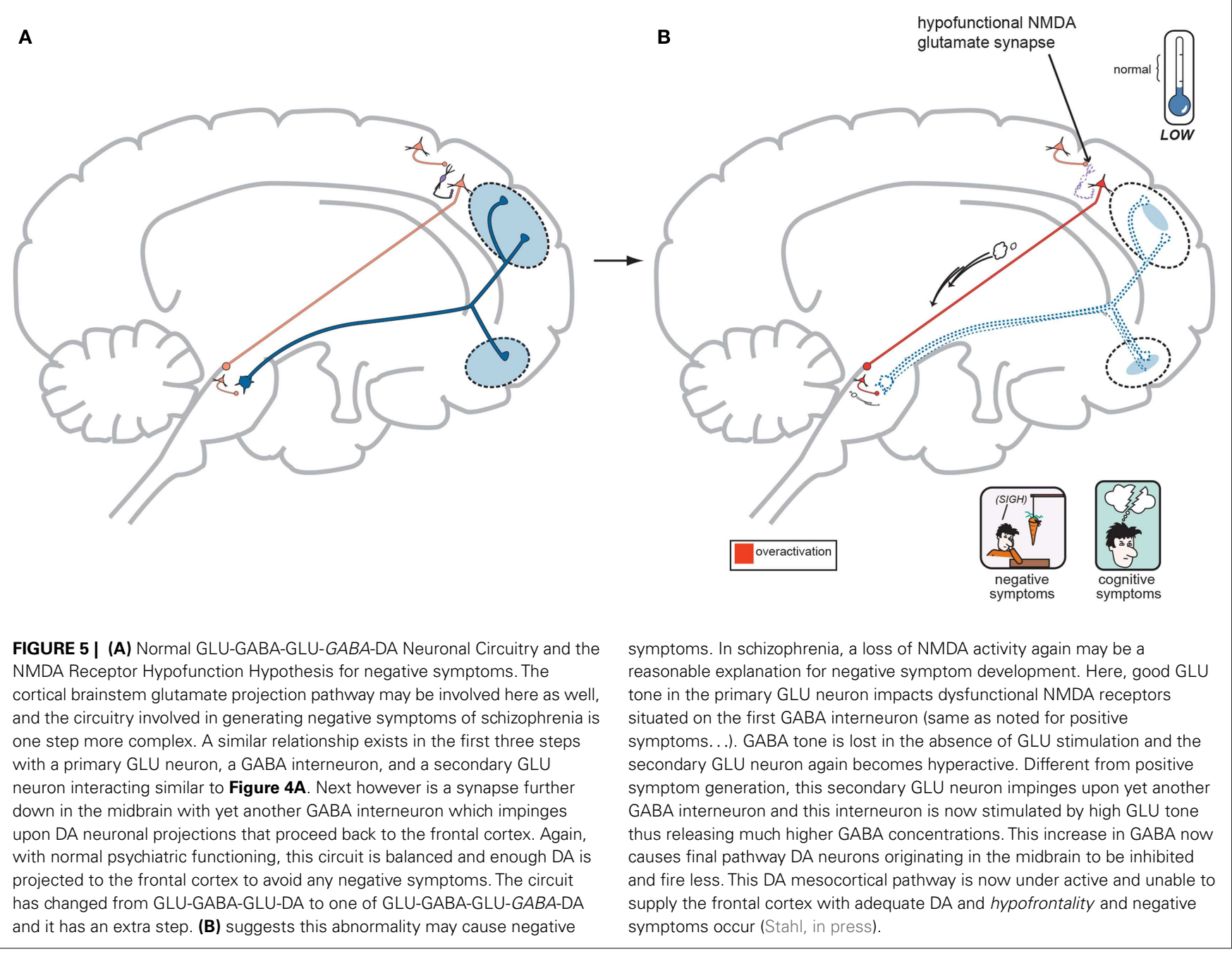




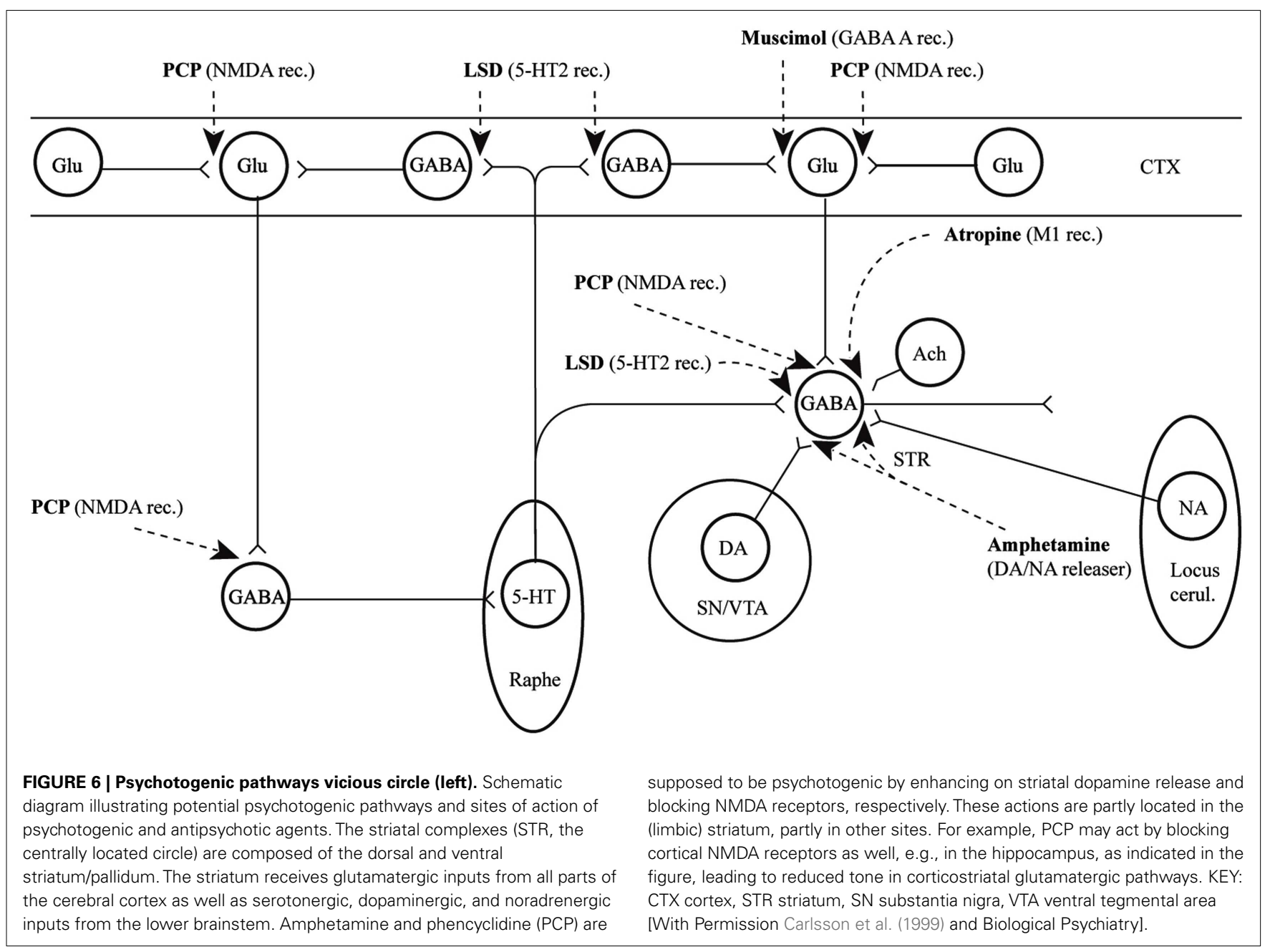

\section{GENETICS PRIMER FOR THE GLUTAMATE PATHWAYS INVOLVED IN SCHIZOPHRENIA}

Accepting that the Dopamine Hypothesis is the final common pathway to schizophrenia symptom development and the complimentary concept that under functioning NMDA receptors or malfunctioning glutamate circuitry is to blame for the ultimate schizophrenia DA abnormalities and symptoms, it now makes sense to better explore how, or why, the GLU system might become dysfunctional in the first place. Again, patients inherit genes that code for proteins. If the gene is mutated or abnormal, then an abnormal protein is created and mass produced. If any of these proteins affect, or are in the vicinity of the GLU pathways, (Figure 3) then the GLU system may falter and change its overall tone. The downstream effect may be to increase DA in the mesolimbic system creating positive symptoms or to lower its activity in the mesocortical symptom allowing for negative symptoms to occur (Stahl, 2007a, 2008).

The NMDA receptor itself is ubiquitous in the CNS and is a key factor in promoting glutamatergic neuronal activity throughout. It is comprised of several small receptor subunits, any of which, if altered by genetic mutation, might alter NMDA activity leading to schizophrenia symptoms. As outlined above, any disruption, making NMDA receptors less active may ultimately account for the genesis of both positive and negative symptoms of schizophrenia (Stahl, 2007b).

NMDA receptors have a well defined three dimensional structure, and they form heterotetramers between two NR1 and two NR2 type subunits. This forms a pore at the center which is a calcium influx channel (Furukawa et al., 2005) that allows neuronal depolarization and activation. NR3 A and B subunits have also been discovered and actually cause NMDA receptors to become less active or insensitive (Sobolevsky et al., 2002). In theory, genetic mutations coding for the protein structure of NR1 or 2 subunits, if making them less sensitive or hypoactive or those making the NR3 A or B subunits more sensitive or more active, might actually dampen NMDA receptor channel opening, allowing less calcium influx. This hyperpolarizing loss of activity might impinge on the GLU-GABA-GLU or GLU-GABA-GLU-GABA circuits outlined previously. The dysfunctional NMDA receptors, sitting on GABA interneurons in this neurocircuitry may allow for dysfunctional downstream DA activity in final common pathways to create positive or negative symptoms respectively.

The gene, GRIN1, codes for the NR1 NMDA receptor subunit and GRIN1 gene abnormalities are a key research interest as 


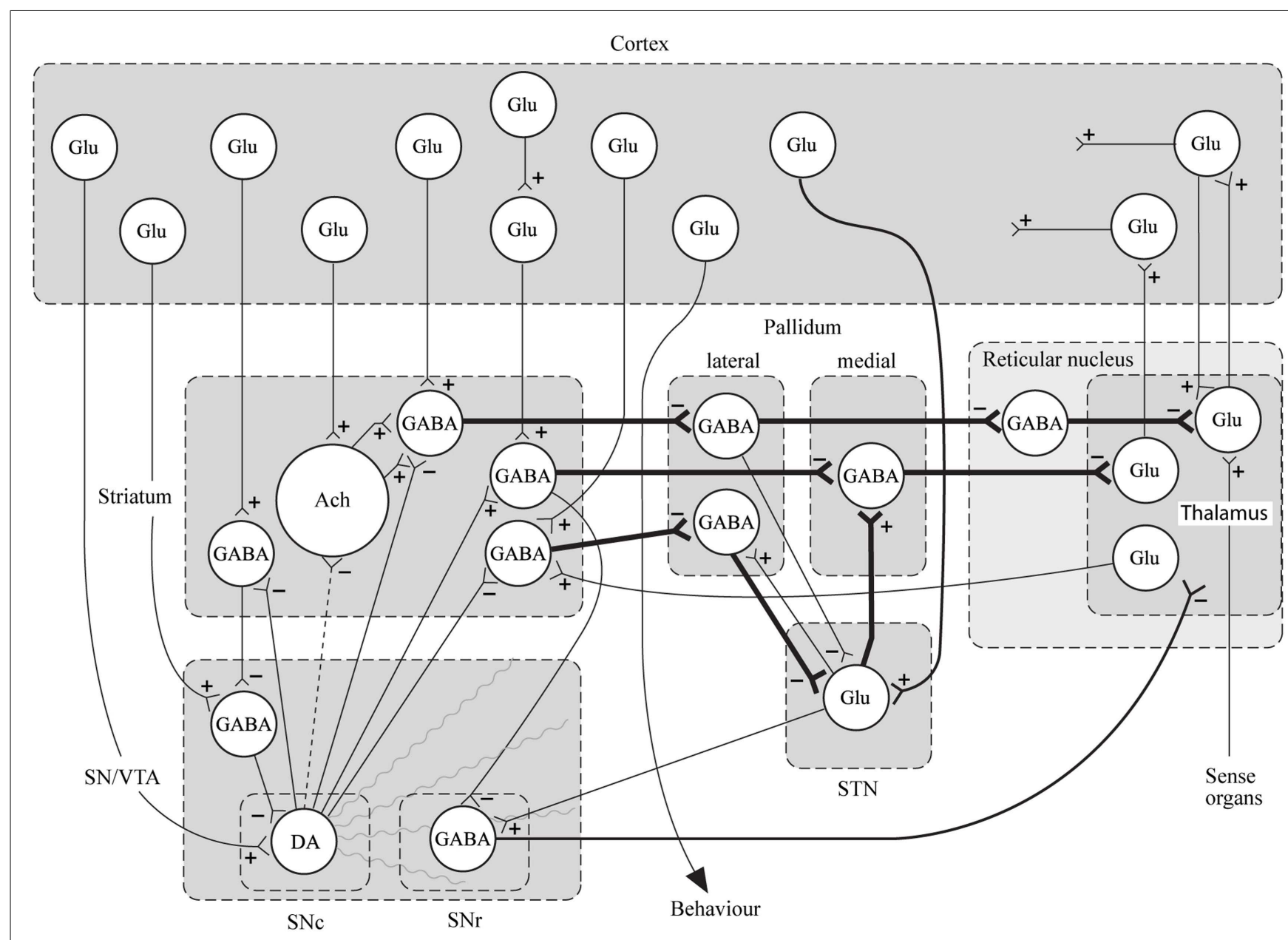

FIGURE 7 | Specific neurocircuitries of the basal ganglia. In this figure the striato-pallido-thalamic pathways are detailed. Among these, the top and bottom pathways drawn with thick lines contain three gabaergic neurons and are referred to as "indirect" pathways. The pathway in between contains two gabaergic neurons and is referred to as "direct." Key: SN, substantia nigra; VTA, ventral tegmental area; STN, subthalamic nucleus; Glu, glutamate; Ach, acetylcholine; DA, dopamine [With Permission Carlsson et al. (1999) and Biological Psychiatry]. a susceptibility gene for schizophrenia. Mice lacking NR1 subunits (gene knock-out mice) exhibit signs that mimic human schizophrenia symptoms (Mohn et al., 1999; Halene et al., 2009). NMDA receptors most often consist of an NR1 subunit (coded by the GRIN1 gene) plus one of the four types of NR2 subunits (GRIN2A, GRIN2B, GRIN2C, and GRIN2D genes). The two subunits of an NMDA receptor may allow for varying functions, as the NR1 subunit possesses the characteristic ion channel properties but the specificity regarding how the NMDA receptor will ultimately function and react is derived from the NR2 subunits housed in the receptor complex. In this manner, NMDA receptors with different functional capacities may be situated in the brain in order to make neurocircuits more, or less active (Moriyoshi et al., 1991; Hollmann and Heinemann, 1994; Nakanishi and Masu, 1994). Summarizing a lengthy evidence base regarding NR1 subunit genetics, there are mixed outcomes showing possible NR1 mutations leading to, or protecting against, the development of schizophrenia. Low NR1 expression due to GRIN1 mutations likely affords low NMDA activity. By re-reviewing Figure 4, notice that these mutations may provide a low level of NMDA activity, and if this occurs at GABA interneurons in the GLU-GABA-GLU circuit, then DA excess and psychotic symptoms may occur if the mutated NMDA receptors reside in the cortical brainstem glutamate projection pathway. Likewise negative symptoms may occur if the cortical brainstem glutamate projections are compromised in the GLU-GABA-GLU-GABA circuit (Figure 5).

GRIN2A is the gene that codes for the NR2A subunit. These often appear preferentially in the frontal cortex and increase in the teen years (Watanabe et al., 1993; Mohrmann et al., 2000), uncanny in a clinical sense, as this age often heralds the onset of schizophrenia. Mice lacking the equivalent of the GRIN2A gene cause abnormal mouse behaviors similar to those observed in animal model schizophrenia (Miyamoto et al., 2001). Literature reviews support a stronger evidence base regarding NMDA receptor hypofunctioning related to abnormal GRIN2A genetic vulnerabilities when compared to GRIN1A. NR2B subunit genetic data suggests mixed results in regards to its conveying risk for schizophrenia. Stronger data may actually support an interaction between these genes, i.e., 
GRIN1A plus GRIN2B, and the interactions between abnormal genes and their resultant proteins may actually convey greater risk for schizophrenia than any single gene alone (Williams et al., 2002; Loftis and Janowsky, 2003; Qin et al., 2005; Tang et al., 2006).

There also exist genes that code for proteins, outside of the NMDA receptor complex that may be involved in the etiology of schizophrenia. These proteins may either directly impact the functioning of the NMDA receptor itself, making it hypoactive in nature, or these proteins may cause a dampening of GLU neuronal activity with the same net effect of allowing terminal DA abnormalities in the GLU-GABA-GLU-DA or GLU-GABA-GLUGABA-DA circuit to cause positive and negative symptoms. For example, a calcineurin gene codes for an NMDA catalytic subunit and rodents missing this gene will have lower (hypofunctioning) NMDA receptor activity and exhibit psychotic model symptoms (Gerber et al., 2003; Miyakawa et al., 2003). A mutated neuregulin1 (NRG1) gene's abnormal protein may also contribute to poor NMDA functioning in rodent and human models (Fischbach and Rosen, 1997; Ozaki et al., 1997; Stefansson et al., 2002, 2003; Hahn et al., 2006). Neuregulin-1 codes for a protein that is required for normal GLU neuron dendritic spine formation. This allows for normal and efficient GLU neurotransmission and synapse formation in the GLU circuits discussed in Figure 3. Poorly functioning neuregulin-1 then would lead to diminished GLU neurotransmission regardless of NMDA receptor capabilities and secondary DA excesses through the GLU-GABA-GLU-DA circuitry yielding positive schizophrenia symptoms.

The NMDA receptor is not the only GLU receptor in the CNS. The non-NMDA ionotropic glutamate receptor kainate-3 gene (GRIK3) codes for glutamate kainite receptors and certain mutations here may pose a possible risk for schizophrenia (Begni et al., 2002). Metabotropic (non-NMDA, non-ligand gated channel) glutamate receptors also exist and their proteins are encoded by the genes GRM2, GRM5, GRM7, and GRM8. Of these, the GRM5 mutation has revealed the most significant results (EmDevon et al., 2001).

Gene $G 72$ codes for a protein that interacts with D-amino acid oxidase enzyme which oxidizes D-serine that usually activates NMDA receptors making them more efficient. G72 mutations might allow for underactive NMDA receptor activity by lowering this enzymatic activity (Chumakov et al., 2002). DTNBP1 (dystrobrevin binding protein 1) codes for dysbindin proteins that are noted to be reduced in the hippocampi and likely frontal lobes of schizophrenia patients. Altered expression of dysbindin likely lowers the activity of lysosome related organelle complexes (BLOC-1) which may lead to abnormal protein distributions in schizophrenia brains. Therefore, dysbindin may influence and cause excitotoxic GLU release increasing neuronal cell death. A reduction in dysbindin activity due to mutations might lead to a hypoactive glutamatergic system as GLU neurons die and are pruned allowing a global GLU hypofunctioning to occur, again regardless of NMDA status (McClintock et al., 2003; Talbot et al., 2004). Akt1 genes code for protein kinase $B$ and are needed for adequate dysbindin functioning. Mutations of Akt1 may subsequently cause poor dysbindin activity and ultimately less glutamatergic tone (Emamian et al., 2004). RGS4 coded proteins are decreased in the prefrontal cortex of schizophrenic patients and mutations may alter $G$ protein mediated signaling via DA, metabotropic GLU, and muscarinic cholinergic receptors. Again, a mutation here does not directly impact NMDA receptors, but would diminish metabotropic GLU receptor activity. This metabotropic input loss, next lowers NMDA receptor activity and lowers GLU tone with the final common pathway being exhibited by DA pathway abnormalities (Chen et al., 2004; Morris et al., 2004). Serine racemase (SRR) enzyme converts $\mathrm{L}$-serine to $\mathrm{D}$-serine, and the latter is a co-agonist of the glycine site of NMDA receptors. NMDA receptors require glycine activity to become fully active, depolarize, and provide optimal neuronal firing. D-Serine also facilitates NMDA firing in a similar manner. Mutations allowing low SRR activity would lower the co-agonist properties, thus lowering the ability of NMDA receptors to be appropriately active. This is another level of potential GLU circuitry hypofunctioning (Morita et al., 2007).

Finally, in the CNS, nitric oxide (NO) has been shown to influence the release of neurotransmitters, learning, memory, and neurodevelopment (Maia de Oliveira et al., 2011) by facilitating neuronal maturation and synaptogenesis. Disturbances in NO release could interfere with both the maturation of cortical neurons and the formation of viable synaptic connections, in accordance with the neurodevelopmental hypothesis of schizophrenia and even during normal synaptic pruning in adolescence and synaptic plasticity into adulthood. Genetically, the promoter region of $\mathrm{NO}$ synthase-I (encoded by the gene NOS1), has been studied in regards to cortical glutamate transmission and been associated with schizophrenia symptoms (Reif et al., 2011). Typically, NOS will generate NO gas, a neurotransmitter, that ultimately functions to enhance GABA interneuron tone and thus lower secondary glutamate neuronal activity. This may protect frontal cortical neurons from excitotoxic destruction. Schizophrenics with specific NOS1 risk alleles (rs41279104 AA/AG) when studied by functional imaging showed slower dorsolateral cortical functioning consistent with hypofrontal negative symptoms. This may occur as this risk gene lowers NOS1 production and secondarily NO levels in the cortex. This causes GABA interneurons to lose tonic firing, lowering of inhibition upon secondary glutamate neurons that may now promote excessive glutamate activity, neuronal destruction, and hypofrontality. Deutsch et al. (1997) conducted a small, open label study using methylene blue to alter the NO pathway and found modest improvements in test subjects with schizophrenia possibly confirming this theoretical model translationally.

All of these aforementioned genes and their protein products do not directly affect NMDA receptor functioning by altering NMDA subunits, but may lower GLU tone in GLU neurons allowing the same downstream effects and schizophrenia symptoms as if the NMDA receptors themselves were hypofunctioning. The above, gene-laden paragraph should lead the reader to the conclusion that there is not one gene that causes schizophrenia but possibly several that may impact the GLU neurocircuitry and if many of these genes are inherited then the patient now has an increased risk to develop schizophrenia. This further alerts that reader that the Dopamine Hypothesis was too simple. It is clear that the D2 receptor antagonists, first and second generation antipsychotics, are modest at best at reducing positive symptoms and very poor at lowering negative symptoms. Schizophrenia development is likely driven by neurodevelopmental, neurodegenerative, and functional 
neurotransmission abnormalities making the etiology of schizophrenia more complex than the dopamine hypothesis even when it is augmented and elaborated by way of the glutamate hypothesis.

In summary, the final common pathway of schizophrenia symptom development embodies the Dopamine Hypothesis where too much limbic DA allows for positive symptoms and too little frontocortical DA allows for negative symptoms. This is likely the tip of the iceberg in that this effect is superficial but may not fully describe or explain what is beneath. The NMDA Receptor Hypofunctioning Hypothesis looks a bit deeper and suggests that faulty NMDA receptors upstream from the DA pathways are the actual cause of the Dopamine Hypothesis. Weak NMDA receptors allow DA excesses to occur in some neurocircuits and deficiencies in others, thus promoting schizophrenia symptoms. Outside of the basic education of these two complimentary explanations regarding the possible etiology of schizophrenia, this paper sought to increase the acceptance of the idea that the NMDA receptors may be key in their own hypothesis, but more globally, any genetic mutation coding for proteins that might impact and cause hypofunctioning anywhere along certain GLU pathways and neurocircuitry may have the same effect as inheriting hypofunctioning NMDA receptors alone. This corollary to the NMDA receptor hypothesis then would suggest that poor glutamatergic tone derived from any source may next lead to the final common pathway of the Dopamine Hypothesis. The next, and final section of this paper will utilize this basic science knowledge and translationally review potential new schizophrenia treatments.

\section{FUTURE TREATMENTS}

\section{DIRECT ACTING NMDA RECEPTOR AGONISTS}

These agents are analogs for endogenous glycine. Using these to increase synaptic concentration of co-agonists for the NMDA receptor should increase NMDA receptor activity. The net effect is greater glutamatergic tone which ideally rectifies the final common pathway of DA neurocircuitry dysfunction. D-Cycloserine is one of the most common agents studied so far and results are equivocal at best. More recently, one of the largest scale, multicenter studies (CONSIST) showed that neither glycine nor D-cycloserine separated from placebo in a randomized trial design in schizophrenia patients suffering from negative symptomatology (Buchanan et al., 2007). Meta-analytically, Tsai and Lin (2010) attempted to evaluate glutamate-based treatments for schizophrenia and analyzed 26 studies meeting descriptive stringent trial design criteria and suggest that GLU manipulating agents can be effective for both positive and negative symptoms. The most robust effect size was noted for treating depressive affective symptoms, negative symptoms, and finally positive symptoms. Full NMDA receptor agonists, glycine and $\mathrm{D}$-serine, appeared to be most effective in these clinical symptom areas but partial agonist, D-cycloserine, was not. All agents were found to be well tolerated.

\section{GLYCINE TRANSPORTER 1 INHIBITORS}

Glycine transporters allow reuptake of glycine and when removed from the synapse, this co-agonist cannot facilitate further NMDA activity. Similar to SSRI for depression, blocking these transporters allows endogenous glycine to increase in synaptic concentration fostering greater NMDA activity to overcome the proposed NMDA receptor hypofunctioning postulate. Sarcosine is an experimental agent with this property. When used as adjunctive therapy with an approved antipsychotic agent, sarcosine has been shown to separate from placebo and has been validated in a few studies (Tsai et al., 2004; Lane et al., 2010). These agents appear to directly improve GLU tone and may have the ability to improve schizophrenia in a manner supporting the NMDA Receptor Hypofunctioning Hypothesis.

\section{KYNURENINE PATHWAY AGENTS}

In the CNS, tryptophan is metabolized and degraded into several metabolites that may inhibit the glutamate neurocircuitry at the level of the NMDA receptor. Specific metabolites of tryptophan in this pathway include: quinolinic acid which is an excitotoxic NMDA receptor agonist, 3-hydroxykynurenine which is a freeradical generator, and kynurenic add (KYNA) which is an antagonist at glutamate receptors. All of these might impair glutamate neurocircuitry and allow for schizophrenia symptoms to develop. Findings in schizophrenia show elevated KYNA which specifically blocks the glycine site on NMDA receptors (Erhardt et al., 2009) and may be more involved in the generation of negative symptoms (cognition, executive dysfunction). Interestingly, KYNA levels increase after infection. Neurodevelopmental theories of schizophrenia postulate that infections in utero or infancy may lend risk to developing schizophrenia. Prospective glycine agonists noted above may be useful if a schizophrenic could be identified as having excesses in KYNA, as competition at the NMDA receptor glycine site may be improved. Translationally, KAT II (Kynurenine aminotransferase: the enzyme responsible for converting tryptophan into KYNA), could be blocked or antagonized in order to lower KYNA levels and improve NMDA functioning. COX-2 inhibitors (antiinflammatory agents) could be the initial or prototypical agents mechanistically as they lower KYNA levels.

\section{mGIUR 2/3 PRESYNAPTIC RECEPTOR AGONISTS}

In some GLU pathways, secondary downstream GLU neurons are actually hyperactive with increased tone. This situation is noted in the NMDA Receptor Hypofunction Hypothesis when the primary GLU neuron is active, but its GABA interneuron has ineffective NMDA receptors and the GABA interneuron will not fire and appropriately inhibit a secondary GLU neuron effectively. This secondary GLU neuron is hyperactive and likely allows DA abnormalities consistent with the Dopamine Hypothesis. Perfectly situated, mGluR $2 / 3$ receptors are typically autoreceptors used to diminish GLU tone. Here, in this specific situation, increasing their tone with an agonist drug would seek to lower the abnormally high GLU firing in these secondary GLU neurons. LY2140023 is an experimental compound that is an $\mathrm{mGluR} 2 / 3$ receptor agonist and was recently studied in schizophrenia in a Phase 2 trial comparing several dose strengths versus an active antipsychotic comparator, olanzapine, and placebo. Neither the experimental drug, nor the approved atypical antipsychotic separated from placebo making interpretation of results negative. The placebo rate was quite high for a schizophrenia study and four subjects suffered seizure-like activity (Kinon et al., 2011). A previous, smaller study versus placebo did show effectiveness in lowering positive and negative symptoms (Patil et al., 2007). 


\section{NEUROPROTECTIVE AGENTS}

Minocycline, an antibiotic, has gained some evidence base in the literature regarding refractory schizophrenia treatment as it may treat negative symptoms. Outside of its antibiotic effects, it is felt to inhibit microglial activation, decrease NO synthase induced apoptosis of neurons, and possibly positively modulate the GluR1 subunits of AMPA glutamate receptors. AMPA receptors were discussed only briefly above, but their dysfunction may also lead to schizophrenia symptoms (Kiss et al., 2011). Glutamate neurocircuitry disruption may occur in the thalamus, more so than the cortex where dysfunctional AMPA receptors reside. AMPA modulators have initial data in human schizophrenia trials suggesting improved long term potentiation rates in hippocampal structures where memory and cognition findings improved secondarily (Wezenberg et al., 2007). Minocycline may have its mechanism of action locally in these non-cortical CNS areas. Therefore, minocycline does not have a direct action on NMDA glutamate receptors, but may influence AMPA receptors that secondarily influence NMDA receptors and improve their activity levels theoretically in schizophrenia (Chaves et al., 2009).

$\mathrm{N}$-acetyl cysteine (NAC) as a prescription is used as a mucolytic agent in infants for respiratory distress but is gaining increased research data and clinical use as a psychotropic. Mechanistically, NAC increases CNS cysteine levels that regulate neuronal intraand extracellular exchange of glutamate through a shared transporter with glutamate. These transporters are mostly located on glial cells where cysteine is taken up and glutamate is released into the extracellular space. Increased free glutamate next may be able to increase the firing of inhibitory metabotropic glutamate receptors resting on glutamate neurons effectively lowering the net, overall release of glutamate (Dean et al., 2011). This may lower NMDA excitotoxicity and preserve balance between

\section{REFERENCES}

Begni, S., Popoli, M., Moraschi, S., Bignotti, S., Tura, G. B., and Gennarelli, M. (2002). Association between the ionotropic gultamate receptor kainate 3 (GRIK3) ser310ala polymorphism and schizophrenia. Mol. Psychiatry 7, 416-418.

Buchanan, R. W., Javitt, D. C., Marder, S. R., Schooler, N. R., Gold, J. M., McMahon, R. P., et al. (2007). The cognitive and negative symptoms in schizophrenia trial (CONSIST): the efficacy of glutamatergic agents for negative symptoms and cognitive impairments. Am. J. Psychiatry 164, 1593-1602.

Carlsson, A., Waters, N., and Carlsson, M. L. (1999). Neurotransmitter interactions in schizophrenia: therapeutic implications. Biol. Psychiatry 46, 1388-1395.

Chaves, C., Marque, C. R., Trzesniak, C., Machado de Sousa, J. P., Zuardi, A. W., Crippa, J. A., et al. (2009). Glutamate-n-methyl-daspartate receptor modulation and minocycline for the treatment of patients with schizophrenia. Braz. J. Med. Biol. Res. 42, 1002-1014.
Chen, X., Dunham, C., Kendler, S., Wang, X., O’Neill, F. A., Walsh, D., et al. (2004). Regulator of G-protein signaling 4 (RGS4) gene is associated with schizophrenia in Irish high density families. Am. J. Med. Genet. B Neuropsychiatr. Genet. 129, 23-26.

Chumakov, I., Blumenfield, M., Guerassimenko, O., Cavarec, L., Palicio, M., Abderrahim, H., et al. (2002). Genetic and physiological data implicating the new human gene G72 and the gene for Dnia. Proc. Natl. Acad. Sci. U.S.A. 99, 13675-13680.

Dean, O., Giorlando, F., and Berk, M. (2011). N-acetylcysteine in psychiatry: current therapeutic evidence and potential mechanisms of action. J. Psychiatry Neurosci. 36, 78-86.

Deutsch, S. I., Rosse, R. B., Schwartz, B. L., Fay-McCarthy, M., Rosenberg, P. B., and Fearing, K. (1997). Methylene blue adjuvant therapy of schizophrenia. Clin. Neuropharmacol. 20, 357-363.

Emamian, E. S., Hall, D., Birnbaum, M. J., Karayiorgou, M., and Gogos, J. amino acid oxidase in schizophre-

the dopamine and glutamate neuropathways. Similar to minocycline, negative schizophrenia symptoms appear to preferentially improve in regards to initial studies' findings.

\section{CONCLUSION}

Clinicians have treated schizophrenia since the 1950s assuming that DA excess in mesolimbic pathways was the cause of positive symptoms and DA deficiencies in the mesocortical pathways allowed for negative symptoms to develop. This Dopamine Hypothesis likely holds true for certain patients suffering from schizophrenia as perhaps these individuals inherited genes for DA receptors, reuptake pumps, metabolic enzymes, etc. that afforded them hyperactivity or hypoactivity in these pathways causing their schizophrenia to develop. If these abnormalities were the only cause or etiology for schizophrenia then currently available antipsychotic agents should be remarkably more effective than they currently are. Educators in psychopharmacology have likely oversimplified their ideas surrounding the pathophysiology of schizophrenia by focusing only on DA mechanisms of illness. The next most robust hypothesis is that discussed in detail above, where hypofunctioning glutamate NMDA receptors are to blame ultimately for DA activity variances which ultimately cause DA based positive or negative symptoms. The authors attempt to convey that focusing only on NMDA receptor hypotheses is also likely too simple. In theory, any genetic mutation that causes a change in functioning of certain GLU neuroanatomic pathways may achieve the same effect as having ineffective NMDA receptors in that abnormal downstream DA activity can occur at several different neuroanatomic sites causing schizophrenia symptoms to develop. Over the next several years, drug compounds focusing more on GLU systems may yield helpful treatments for schizophrenia patients.

A. (2004). Convergent evidence for impaired AKT1- GSK3beta signaling in schizophrenia. Nat. Genet. 36, 131-137.

EmDevon, R. S., Anderson, S., and Teague, P. W. (2001). The genomic organization of the metabotropic glutamate receptor subtype 5 gene, and its association with schizophrenia. Mol. Psychiatry 6, 311-314.

Erhardt, S., Olsson, S. K., and Engberg, G. (2009). Pharmacological manipulation of kynurenic acid: potential in the treatment of psychiatric disorders. CNS Drugs 23, 91-101.

Fischbach, G. D., and Rosen, K. M. (1997). ARIA: a neuromuscular junction neuregulin. Annu. Rev. Neurosci. 20, 429-458.

Furukawa, H., Singh, S. K., Mancusso, R., and Gouaux, E. (2005). Subunit arrangement and function in NMDA receptors. Nature 438, 185-192.

Gerber, D. J., Hall, D., Miyakawa, T. Demars, S., Gogos, J. A., Karayiorgou, M., et al. (2003). Evidence for association of schizophrenia with genetic variation in the $8 \mathrm{p} 213$ gene, PPP3CC, encoding the calcineurin gamma subunit. Proc. Natl. Acad. Sci. U.S.A. 100, 8993-8998.

Hahn, C. G., Wang, H. Y., Cho, D. S., Talbot, K., Gur, R. E., Berrettini, W. H., et al. (2006). Altered neuregulin I-erbB4 signaling contributes to NMDA receptor hypofunction in schizophrenia. Nat. Med. 12, 824-828.

Halene, T. B., Ehrlichman, R. S., Liang, Y., Christian, E. P., Jonak, G. J., Gur, T. L., et al. (2009). Assessment of NMDA receptor NR1 subunit hypofunction in mice as a model for schizophrenia. Genes Brain Behav. 8 , 661-675.

Hollmann, M., and Heinemann, S. (1994). Cloned glutamate receptors. Annu. Rev. Neurosci. 17, 31-108.

Kinon, B. J., Zhang, L., Millen, B. A., Osuntokun, O. O., Williams, J. E., Kollack-Walker, S., et al. (2011). A multicenter, inpatient, phase 2, double-blind, placebocontrolled dose-ranging study of LY2140023 monohydrate in patients with DSM-IV schizophrenia. J. Clin. Psychiatry 31, 349-355. 
Kiss, T., Hoffmann, W. E., Scott, L., Kawabe, T. T., Milici, A. J., Nilsen, E. A., et al. (2011). Role of thalamic projection in NMDA receptor-induced disruption of cortical slow oscillation and short-term plasticity. Front. Psychiatry 4:14. doi:10.3389/fpsyt.2011.00014

Lane, H. Y., Lin, C. H., Huang, Y. J., Liao, C. H., Chang, Y. C., Tsai, G. E., et al. (2010). A randomized, double-blind, placebocontrolled comparison study of sarcosine (N-methylglycine) and $\mathrm{D}$ serine add-on treatment for schizophrenia. Int. J. Neuropsychopharmacol. 13, 451-460.

Loftis, J. M., and Janowsky, A. (2003). The N-methyl-D-aspartate receptor subunit NR2B: localization, functional properties, regulation, and clinical implications. Pharmacol. Ther. 97, 55-85.

Maia de Oliveira, J. P., Lobão, B., Machado-de-Sousa, J. P., Baker, G. B., Dursun, S., and Hallak, J. E. C. (2011). Targeting the NMDA receptor nitric oxide-cyclic GMP pathway to develop non-dopaminergic antipsychotic medications for schizophrenia. Rev. Bras. Psiquiatr. 33, 223-224.

McClintock, W., Shannon Weickert, C., Halim, N. D., Lipska, B. K., Hyde, T. M., Herman, M. M., et al. (2003). Reduced Expression of Dysbindin Protein in the Dorso-Lateral Prefrontal Cortex of Patients with Schizophrenia. Program No. 317.9. 2003 Abstract Viewer/Itinerary Planner. Washington, DC: Society for Neuroscience.

Meltzer, H. Y., and Stahl, S. M. (1976). The dopamine hypothesis of schizophrenia: a review. Schizophr. Bull. 2, 19-76.

Miyakawa, T., Leiter, L. M., Gerber, D. J., Gainetdinov, R. R., Sotnikova, T. D., Zeng, H., et al. (2003). Conditional calcineurin knockout mice exhibit multiple abnormal behaviors related to schizophrenia. Proc. Natl. Acad. Sci. U.S.A. 100, 8987-8992.

Miyamoto, Y., Yamada, K., Noda, Y., Mori, H., Mishina, M., Nabeshima, T., et al. (2001). Hyperfunction of dopaminergic and serotonergic neuronal systems in micelacking the NMDA receptor epsilon 1 subunit. J. Neurosci. 21, 750-757.

Mohn, A. R., Gainetdinov, R. R., Caron, M. G., and Koller, B. H. (1999). Mice with reduced NMDA receptor expression display behaviors related to schizophrenia. Cell 98, 427-436.

Mohrmann, R., Hatt, H., and Gottmann, K. (2000). Developmental regulation of subunit composition of extrasynaptic
NMDA receptors in neocortical neurons. Neuroreport 11, 1203-1208.

Morita, Y., Ujike, H., Tanaka, Y., Otani, K., Kishimoto, M., Morio, A., et al. (2007). A genetic variant of the serine racemase gene is associated with schizophrenia. Biol. Psychiatry 61, 1200-1203.

Moriyoshi, K., Masu, M., Ishii, T., Shigemoto, R., Mizuno, N., and Nakanishi, S. (1991). Molecular cloning and characterization of the rat NMDA receptor. Nature 354, 31-37. Morris, D. W., Rodgers, A., McGhee, K. A., Schwaiger, S., Scully, P. Quinn, J., et al. (2004). Confirming RGS4 as a susceptibility gene for schizophrenia. Am. J. Med. Genet. B Neuropsychiatr. Genet. 125, 50-53.

Nakanishi, S., and Masu, M. (1994). Molecular diversity and functions of glutamate receptors. Аnnu. Rev. Biophys. Biomol. Struct. 23, 319-348.

Opler, L. A., and Opler, M. G. (2012). "Antipsychotics: where have we been? Where are we now? Where are we going?", in Antipsychotic Drugs: Pharmacology, Side Effects and Abuse Prevention, eds T. L. Schwartz, J. Megna, and M. Topel (New York: Nova Scientific Press) (in press).

Ozaki, M., Sasner, M., Yano, R., Lu, H. S., and Buonanno, A. (1997). Neuregulin-beta induces expression of an NMDA-receptor subunit. Nature 390, 691-694.

Patil, S. T., Zhang, L., Martenyi, F., Lowe, S. L., Jackson, K. A., Andreev, B. V., et al. (2007). Activation of mGlu2/3 receptors as a new approach to treat schizophrenia: a randomized phase 2 clinical trial. Nat. Med. 13, 1102-1107.

Qin, S., Zhao, X., Pan, Y., Liu, J., Feng, G., Fu, J., et al. (2005). An association study of the N-methyl-Daspartate receptor NR1 subunit gene (GRIN1) and NR2B subunit gene (GRIN2B) in schizophrenia with universal DNA microarray. Eur. J. Hum. Genet. 13, 807-814.

Reif, A., Schecklmann, M., Eirich, E., Jacob, C. P., Jarczok, T. A., KittelSchneider, S., et al. (2011). A functional promoter polymorphism of neuronal nitric oxide synthase moderates prefrontal functioning in schizophrenia. Int. J. Neuropsychopharmacol. 14, 887-897.

Sadock, B. J., and Sadock, V. (2003). Kaplan and Sadock's Synopsis of Psychiatry, 9th Edn. Philadelphia: Lippincott Williams and Wilkins.

Schwartz, T. L., Sachdeva, S., and Stahl, S. M. (2012). Genetic data supporting the NMDA glutamate receptor hypothesis for schizophrenia. Curr. Pharm. Des. 18, 1580-1592.

Sobolevsky, A. I., Rooney, L., and Wollmuth, L. P. (2002). Staggering of subunits in NMDAR channels. Biophys. J. 83, 3304-3314.

Stahl, S. M. (2007a). Beyond the dopamine hypothesis to the NMDA glutamate receptor hypofunction hypothesis of schizophrenia. CNS Spectr. 12, 265-268.

Stahl, S. M. (2007b). The genetics of schizophrenia converge upon the NMDA glutamate receptor. CNS Spectr. 12, 583-588.

Stahl, S. M. (2008). Essential Psychopharmacology, 3rd Edn. New York, NY: Cambridge University Press.

Stahl, S. M. (in press). Essential Psychopharmacology: Neuroscientific Basis and Practical Applications, 4th Edn. Cambridge University Press.

Stefansson, H., Sarginson, J., Kong, A., Yates, P., Steinthorsdottir, V. Gudfinnsson, E., et al. (2003). Association of neuregulin-1 with schizophrenia confirmed in a Scottish population. Am. J. Hum. Genet. 72, 83-87.

Stefansson, H., Sigurdsson, E. Steinthorsdottir, V., Bjornsdottir, S., Sigmundsson, T., Ghosh, S., et al. (2002). Neuregulin-1 and susceptibility to schizophrenia. Am. J. Hum. Genet. 71, 877-892.

Straub, R. E., and Weinberger, D. R. (2006). Schizophrenia genes: famine to feast. Biol. Psychiatry 60 81-83.

Talbot, K., Eidem, W. L., Tinsley, C. L., Benson, M. A., Thompson, E. W. Smith, R. J., et al. (2004). Dysbindin1 is reduced in intrinsic, glutamatergic terminals of the hippocampal formation in schizophrenia. J. Clin. Invest. 113, 1353-1363.

Tang, J., Chen, X., Xu, X., Wu, R., Zhao, J., Hu, Z., et al. (2006). Significant linkage and association between a functional (GT)n polymorphism in promoter of the N-methyl-d-aspartate receptor subunit gene (GRIN2A) and schizophrenia. Neurosci. Lett. 409 , 80-88.

Tsai, G., Lane, H. Y., Yang, P., Chong, M. Y., and Lange, N. (2004) Glycine transporter I inhibitor, Nmethylglycine (sarcosine), added to antipsychotics for the treatment of schizophrenia. Biol. Psychiatry 55, 452-456.

Tsai, G. E., and Lin, P. Y. (2010). Strategies to enhance n-methyl-daspartate receptor-mediated neurotransmission in schizophrenia, a critical review and meta-analysis. Curr. Phar. Des. 16, 522-537.
Watanabe, M., Inoue, Y., Sakimura, K., and Mishina, M. (1993). Distinct spatio-temporal distributions of the NMDA receptor channel subunit mRNAs in the brain. Ann. N. Y. Acad. Sci. 707, 463-466.

Wezenberg, E., Verkes, R. J., Ruigt, G. S., Hulstijn, W., and Sabbe, B. G. (2007). Acute effects of the ampakine farampator on memory and information processing in healthy elderly volunteers. Neuropsychopharmacology 32, 1272-1283.

Williams, N. M., Bowen, T., Spurlock, G., Norton, N., Williams, H. J., Hoogendoorn, B., et al. (2002). Determination of the genomic structure and mutation screening in schizophrenic individuals for five subunits of the N-methyl-Daspartate glutamate receptor. $\mathrm{Mol}$. Psychiatry 7, 508-514.

Conflict of Interest Statement: Drs. Schwartz and Sachdeva have no known conflicts of interest regarding any products outlined in this paper. Dr. Stahl has served as a Consultant to Abbott, Advent, Alkermes, Arena, Astra Zeneca, BioMarin, Boehringer Ingelheim, Bristol Myers Squibb, Cypress Bioscience, Dainippon Sumitomo, Eli Lilly, Forest, Genomind, Janssen, Jazz, LaboPharm, Lundbeck, Merck, Neuronetics, Novartis, ONO, Orexigen, Otsuka, PamLabs, PGxHealth, Pfizer, Rexahn, Royalty Pharma, Schering Plough, Servier, Shire, Valeant, and Vivus. He has served on speakers bureaus for Merck, PamLabs, Dainippon Sumitomo/Sepracor/Sunovion, Eli Lilly, and has received research and/or grant support from Astra Zeneca, Biomarin, Dainippon Sumitomo/Sepracor/Sunovion, Eli Lilly, Forest, Genomind, Merck/Schering Plough, PamLabs, Pfizer, PGxHealth, Servier, Shire, Torrent, and Trovis.

Received: 21 August 2012; accepted: 31 October 2012; published online: 26 November 2012.

Citation: Schwartz TL, Sachdeva S and Stahl SM (2012) Glutamate neurocircuitry: theoretical underpinnings in schizophrenia. Front. Pharmacol. 3:195. doi: 10.3389/fphar.2012.00195

This article was submitted to Frontiers in Neuropharmacology, a specialty of Frontiers in Pharmacology.

Copyright (C) 2012 Schwartz, Sachdeva and Stahl. This is an open-access article distributed under the terms of the Creative Commons Attribution License, which permits use, distribution and reproduction in other forums, provided the original authors and source are credited and subject to any copyright notices concerning any third-party graphics etc. 